\title{
El papel de la quimioterapia en el tratamiento paliativo integrado del cáncer
}

Toda actuación médica debería estar impregnada de una idea expresada ya en el siglo XVI de «curar a veces, mejorar a menudo, cuidar siempre». Basándonos en esta idea, la Oncología se plantea como primer objetivo la curación de la enfermedad y, si esto no es posible, el incremento de la supervivencia. Ambos objetivos siempre deberán acompañarse de un alivio sintomático y un intento de mejorar la calidad de vida, aspectos englobados dentro de la Medicina Paliativa. Por tanto, esta última debe conjugarse con la Medicina Curativa desde el comienzo de la enfermedad, e irá cobrando cada vez mayor importancia a medida que se produzca una progresión de la misma. El tratamiento paliativo del cáncer englobaría desde la prevención de la toxicidad producida por el tratamiento activo, pasando por el tratamiento de soporte $\mathrm{y}$ apoyo al paciente en cualquier fase evolutiva, hasta llegar a los llamados «cuidados paliativos» del enfermo terminal ${ }^{1}$.

Hoy día las neoplasias sólidas metastásicas en la gran mayoría de los casos son incurables, aunque se puede lograr una supervivencia prolongada cuando existe un número reducido de metástasis que pueden ser resecadas. De esta manera en el cáncer avanzado los objetivos principales del tratamiento serán el aumento de la supervivencia y la mejoría de la calidad de vida. Los enfermos con neoplasias diseminadas habitualmente presentan una sintomatología diversa, acompañada o no de trastornos emocionales, problemas económicos y/o socio-familiares. A ello hay que añadir la toxicidad y las secuelas que podemos producir con el tratamiento activo ${ }^{1}$.

Por tanto, el tratamiento paliativo del cáncer avanzado requiere un enfoque integrado, como bien indica el doctor Camps en este mismo número, que engloba a numerosas especialidades: Oncología Médica, Radioterapia, Cirugía, Atención Primaria, Psiquiatría y Psicología, Unidades del Dolor y Unidades de Cuidados Paliativos (hospitalarias y domiciliarias) ${ }^{2,3}$. Así, desde el diagnóstico de la enfermedad, el tratamiento deberá cubrir los siguientes puntos:

1. Aliviar toda la sintomatología presente.

2. Atender otros factores que puedan influir en el estado general: emocionales, sociales, espirituales, etc.
3. Valorar la posibilidad de iniciar un tratamiento activo: quimioterápico fundamentalmente, y en algunos casos quirúrgico o radioterápico.

La quimioterapia paliativa ha mostrado un claro beneficio, tanto en supervivencia como en calidad de vida, en algunos tumores como el cáncer de mama y el cáncer colorrectal. En otros, aunque no mejore en gran medida la supervivencia sí mejora la calidad de vida, como es el caso del cáncer de pulmón no microcítico y el cáncer de páncreas.

Para lograr el máximo beneficio de la quimioterapia debemos de tratar de optimizar su administración. Actualmente conocemos alguna manera de conseguirlo, pero otras todavía están pendientes de esclarecerse. Por ejemplo, hoy se sabe que la edad no debe ser una limitación para el tratamiento con quimioterapia, sino que dependerá del estado general y de la comorbilidad ${ }^{4}$. Durante todo tratamiento antineoplásico se deberán establecer unas medidas de soporte adecuadas; además del tratamiento sintomático para la enfermedad es preciso administrar los antieméticos indicados en cada caso, así como pautar eritropoyetina en el caso de detectar una anemia inducida por la quimioterapia. Con ello evitaremos un deterioro del enfermo y posibles ingresos hospitalarios durante el tratamiento activo, que limitarían la posible mejoría en la calidad de vida.

Sin embargo, como ya se ha comentado, existen todavía dudas sobre la manera más óptima de administrar la quimioterapia. La duración del tratamiento adyuvante está más o menos establecida (entre 4 y 6 ciclos en la mayoría de los tumores), pero en la enfermedad avanzada se desconoce si el tratamiento mantenido hasta la progresión podría tener un mayor beneficio que un número limitado de ciclos. El doctor Aparicio realiza una excelente revisión sobre esta controversia, y, como explica en su artículo, el beneficio es probable que dependa de cada tumor y de cada enfermo ${ }^{5}$. En líneas generales parece que las enfermas con cáncer de mama serían las que más se podrían beneficiar de un tratamiento prolongado. Es posible que también algunos enfermos con cáncer colorrectal obtuvieran un beneficio con esta medida, aunque los resultados de distintos estudios 
son contradictorios. Sin haber mostrado una clara mejoría de la supervivencia, el tratamiento mantenido podría inducir una toxicidad acumulada que terminase por empeorar la calidad de vida. Por tanto, lo más prudente sería individualizar cada caso y tomar una decisión conjuntamente con cada enfermo.

Otro tema conflictivo con el que nos enfrentamos los oncólogos en el día a día es la decisión sobre el número de líneas de quimioterapia que se deberían administrar. Esto, evidentemente, depende del tipo de neoplasia. En algunos casos (cáncer de mama, cáncer colorrectal, cáncer de ovario, cáncer de pulmón, etc.) se ha demostrado el beneficio de la administración de 2 o incluso más líneas. Pero con frecuencia nos encontramos ante neoplasias en progresión que han sido refractarias a más de una línea de quimioterapia, en las que un nuevo tratamiento quimioterápico tendría una indicación muy dudosa, que incluso podría contribuir a un mayor deterioro del enfermo. Por ello en estas situaciones, a pesar de la frecuente demanda de tratamiento activo por parte del paciente y la familia, debemos evaluar cuidadosamente en cada caso el riesgocoste-beneficio que tendría una nueva línea de quimioterapia y priorizar ante todo el tratamiento de soporte.

\section{Bibliografía}

1. González Barón M. Conceptos de Medicina Paliativa. Tratamientos de soporte en Oncología. Papel del médico de familia en la Medicina Paliativa oncológica. En: González Barón M, Ordóñez A, Feliu J, et al, editores. Tratado de Medicina Paliativa y tratamiento de soporte en el enfermo con cáncer. Madrid: Editorial Médica Panamericana; 1996; 1. p. 1-12.

2. Camps Herrero C, Martínez Banaclocha N. Una nueva concepción de los cuidados paliativos en oncología: los cuidados continuos y la integración de niveles. Rev Oncol 2002.;4:476-84.

3. Señor de Uría C, Mengual Vich A, Santamaría Semis J. Desarrollo de los cuidados paliativos. El equipo multidisciplinario. En: González Barón M, et al, editores. Tratado de Medicina Paliativa y tratamiento de soporte en el enfermo con cáncer. Madrid: Editorial Médica Panamericana; 1996;7. p. 85-96.

4. González Barón M, Casado Sáenz E. Cáncer y vejez. En: González Barón M, González Montalvo JI, Feliu Batlle J, et al, editores. Cáncer en el anciano. Barcelona: Masson; 2001;1. p. 1-13.

5. Aparicio Urtasun J, Díaz Beveridge R. ¿Cuál es la duración óptima de la quimioterapia paliativa en los pacientes con cáncer avanzado? Rev Oncol 2002;4:471-5.

Manuel González Barón Servicio de Oncología Médica. Hospital Universitario La Paz. Universidad Autónoma. Madrid. 